$4^{\text {th }}$ Micro and Nano Flows Conference

UCL, London, UK, 7-10 September 2014

\title{
Off-plane motion of an oblate capsule in a simple shear flow.
}

\author{
Anne-Virginie SALSAC ${ }^{1, *}$, Claire DUPONT ${ }^{1,2}$, Fabien DELAHAYE $^{1}$, Dominique BARTHES-BIESEL $^{1}$ \\ ${ }^{*}$ Corresponding author: Tel.: +33 (0)3 442373 38; Email: a.salsac@utc.fr \\ ${ }^{1}$ Biomechanics and Bioengineering Laboratory, UMR CNRS 7338, \\ Université de Technologie de Compiègne, France \\ ${ }^{2}$ Solids Mechanics Laboratory, UMR CNRS 7649, Ecole Polytechnique, France
}

\begin{abstract}
We investigate the mechanical equilibrium state of an oblate capsule when its revolution axis is initially off the shear plane. We consider an oblate capsule with an aspect ratio of 0.5 and a strain-hardening membrane. The three-dimensional fluid-structure interaction problem is solved numerically by coupling a finite element method with a boundary integral method. The capsule converges towards the same mechanical equilibrium state whatever the initial orientation. This equilibrium depends on the capillary number $C a$, which compares the viscous to the elastic forces and on the viscosity ratio $\lambda$ between the internal and external fluids. For $\lambda=1$, the tumbling and swinging motions, observed when the revolution axis is initially in the shear plane, are mechanically stable until $C a \sim 1$; when $C a$ is further increased, the capsule assumes the rolling motion that is observed when its revolution axis is initially aligned with the vorticity axis. When $\lambda$ is increased, the tumbling-to-swinging transition appears for higher $C a$ and the swinging-to-rolling transition for lower $C a$. For $\lambda \geq 5$, the swinging regime completely disappears: depending on $C a$, it is then either the tumbling or the rolling motion that is the mechanical equilibrium state.
\end{abstract}

Keywords: oblate capsule, finite element method, boundary integral method, off plane motion

\section{Introduction}

Microcapsules consist of a liquid internal medium protected within a thin membrane. The dynamics of a spheroidal capsule in simple shear flow has recently received a lot of attention (Sui et al., 2008; Walter et al., 2011; Dupont et al., 2013), owing to its relevance to the motion of a red blood cell. Most studies have modelled the capsule with the revolution axis in the shear plane, which is a special case, as it is an equilibrium configuration in Stokes flow conditions. It has then been shown that the capsule motion depends on the capillary number $C a$, ratio of the viscous to elastic forces. At low $\mathrm{Ca}$, the capsule has a tumbling motion and rotates like a solid particle. At higher values of $C a$, the capsule experiences a transition where two of its in-plane major axes are almost equal. Then as $C a$ is further increased, the capsule takes a swinging motion where it oscillates about the straining direction, while the membrane rotates around the deformed shape (fluidlike motion).

The off-plane motion of a prolate capsule in shear flow has been recently studied (Dupont et al., 2013; Cordasco and Bagchi, 2013). It is found that the low $\mathrm{Ca}$ tumbling motion in the shear plane is mechanically unstable. Indeed, for any initial orientation, the capsule places its revolution axis along the vorticity axis perpendicularly to the shear plane (rolling regime). As $\mathrm{Ca}$ is increased, the capsule longest axis moves away from the vorticity axis and precesses around it. At still higher values of $C a$, the capsule longest axis goes to the shear plane where a stable swinging regime is observed. Dupont et al. (2013) have shown that those stable equilibrium states do not depend on the initial orientation of the capsule revolution axis relative to the shear plane. 
The case of oblate capsules has been considered by Cordasco and Bagchi (2013), who find that at moderate $C a$, the capsule tends to place its short axis in the shear plane where it takes different motions from tumbling, kayaking to swinging as a function of $\mathrm{Ca}$ and aspect ratio. However, as an oblate capsule takes quite a long time to reach equilibrium, most of the situations considered by Cordasco and Bagchi (2013) were not time converged. Omori et al. (2012) have also simulated the motion of an oblate capsule with a strain-softening neoHookean membrane and with short-to-long axis ratio equal to 0.6. However, they have only considered two capillary numbers $(\mathrm{Ca}=0.3$ and 1.0) and have not studied the stability of the tumbling motion, which takes place at low $\mathrm{Ca}$.

It follows that a thorough parametric study of the dynamics of oblate capsules is needed to fully understand its behavior. The objective of the paper is to investigate the influence of the capillary number, viscosity ratio and initial orientation on the equilibrium configurations of an oblate capsule until an equilibrium state is reached.

\section{Method}

We consider a capsule, which is oblate in its reference undeformed state with aspect ratio $a / b=0.5$, where $2 a$ denotes the length of the revolution axis and $2 b$ the length of the two orthogonal axes. We choose as length scale $\ell=$ $\left(a b^{2}\right)^{1 / 3}$, the radius of the sphere with the same volume as the capsule. The capsule is filled with a Newtonian incompressible fluid with viscosity $\lambda \mu$ and suspended in an unbounded Newtonian incompressible fluid with viscosity $\mu$, where $\lambda$ is the internal to external viscosity ratio. The internal and surrounding fluid have the same density, thus excluding gravity effect. The Reynolds number of the flow is assumed to be very small, so that the internal and external flows are governed by the Stokes equations. The capsule is subjected to a simple shear flow with shear rate $\dot{\gamma}$ : the base flow velocity is

$$
\mathbf{v}^{\infty}=\dot{\gamma} y \mathbf{e}_{x}
$$

in the laboratory reference frame. The capsule and flow centers $O$ are identical.
At time $\dot{\gamma} t=0$, the capsule revolution axis makes an angle $\zeta_{0}$ with the vorticity axis and its projection in the shear plane is aligned with the $x$-axis (Fig. 1).

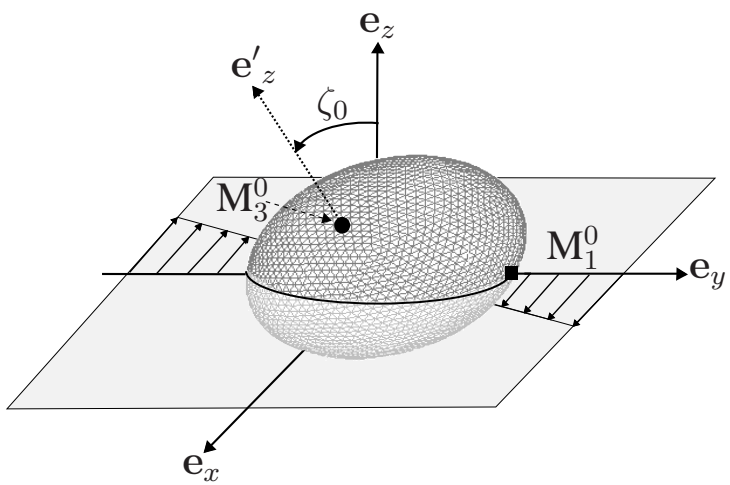

Fig. 1: Reference configuration of the oblate capsule subjected to a simple shear flow at $\dot{\gamma} t=$ 0 . The initial capsule orientation is given by $\zeta_{0}$, the angle between the capsule revolution axis $\mathbf{e}_{z}^{\prime}$ and the flow vorticity axis $\mathbf{e}_{z}$.

The capsule has a very thin membrane, modeled as an isotropic hyperelastic surface with shear modulus $G_{s}$ and area dilatation modulus $K_{s}$. The bending resistance of the capsule membrane is neglected. We consider that the capsule membrane behaviour is described by the Skalak law, a strain-hardening constitutive law, initially proposed by Skalak et al. (1973) to model the red blood cell membrane. In this case, the principal Cauchy elastic tensions $\tau_{1}$ and $\tau_{2}$ are given in terms of the in-plane principal stretch ratios $\lambda_{1}$ and $\lambda_{2}$ by

$$
\tau_{1}=\frac{G_{s}}{\lambda_{1} \lambda_{2}}\left[\lambda_{1}^{2}\left(\lambda_{1}^{2}-1\right)+C\left(\lambda_{1} \lambda_{2}\right)^{2}\left(\left(\lambda_{1} \lambda_{2}\right)^{2}-1\right)\right] .
$$

with the corresponding expression for $\tau_{2}$. The surface shear modulus $G_{s}$ and area dilatation modulus $K_{s}$ are related by $K_{s}=G_{s}(1+2 C)$, where $C$ is a constant such that $C>-1 / 2$. The capsule motion and deformation are thus governed by the membrane constitutive law, the ratio $K_{s} / G_{s}$, the capsule initial orientation $\zeta_{0}$, the capillary number $C a=\mu \dot{\gamma} \ell / G_{s}$, which measures the ratio between the viscous and the elastic forces and the viscosity ratio $\lambda$ between the internal and surrounding fluids. In this study, we consider $C=1$ and we focus on the influence of $\zeta_{0}, C a$ and $\lambda$ on the capsule dynamics. 
The problem is solved numerically by coupling a boundary integral technique to compute the fluid flows (inside and outside the capsule) to a finite element method (for the mechanics of the capsule wall). A Lagrangian tracking of the position of the membrane material points is performed over time (Walter et al., 2010; Foessel et al., 2011). One of the advantages of this numerical method is that all the problem unknown quantities need to be determined on the capsule surface only. The latter is meshed by subdividing sequentially the 20 triangular faces of an icosahedron inscribed in a sphere until the desired number of elements is reached. Nodes are then added at the middle of all the element edges and projected onto the sphere in order to generate second order $\left(P_{2}\right)$ elements. This mesh is then deformed into an ellipsoidal mesh with the desired axis ratio. Here we use a mesh with 2562 nodes and 1280 triangular curved elements. The numerical method is stable when the time step satisfies the condition $\dot{\gamma} \triangle t<O(h C a)$, where $h$ is the typical nondimensional mesh size (Walter et al., 2010). We use $\dot{\gamma} \triangle t=5 \times 10^{-3}$ for $C a \geq 0.5$ and decrease the time step proportionally for lower $C a$.

The global geometry of the capsule is evaluated by means of the ellipsoid of inertia of the deformed shape. We denote $L_{i}$ the half lengths of the principal axes of the ellipsoid of inertia $\left(L_{1}>L_{2}>L_{3}\right)$. The capsule global motion is measured from the position of the short axis tip $M_{3}^{t}$ at time $t$, computed from the ellipsoid of inertia at each time step. The membrane rotation is measured from the motion of the point $M_{3}^{0}$, a specific mesh node initially located on the capsule short axis (Fig. 1). In order to identify clearly the motion of the short axis in space, we introduce the angle $\bar{\zeta}$ between the capsule short axis $O M_{3}^{t}$ and the shear plane.

\section{Results and discussion}

As for prolate capsules (Dupont et al., 2013), we find that the capsule converges towards a unique equilibrium motion, which does not depend on the initial orientation $\left.\zeta_{0} \in\right] 0^{\circ}, 90^{\circ}$. For example, Fig. 2 shows, for $C a=0.01$, that the angle $\bar{\zeta}$ tends to zero for three different initial positions $\zeta_{0}$. The equilibrium motion of the cap- sule then depends only on the capillary number $C a$ and viscosity ratio $\lambda$. We first focus on the equilibrium states which are obtained for $\lambda=1$.

For small to moderate flow strengths corresponding to $\mathrm{Ca}<0.9$, the short axis migrates towards the shear plane for any initial orientation $\zeta_{0}$ (Fig. 2, 3). Some oscillations of $\zeta$ are still observed in Fig. 2 at equilibrium state. Although they do not completely disappear, they remain of very small amplitude. For $C a=0.01$, it is difficult to determine precisely which axis corresponds to the short axis at equilibrium, as $L_{2} \approx L_{3}$. Like Omori et al. (2012), we therefore also find that the point $M_{3}^{0}$ migrates to the shear plane. It follows that the situation is identical to the one considered by Walter et al. (2011) where the capsule revolution axis is initially positioned in the shear plane $\left(\zeta_{0}=90^{\circ}\right)$. Walter et al. (2011) find that at low $\mathrm{Ca}(\mathrm{Ca}<0.02)$, the capsule takes a quasisolid tumbling motion. For $C a \in[0.02,0.05]$, the capsule has a transition motion: it takes a nearly isotropic shape in the shear plane with nearly equal in-plane principal axes. For higher values of $\mathrm{Ca}(\mathrm{Ca}>0.5)$, the capsule takes a swinging motion, where the axis $O M_{3}^{t}$ has a slightly varying length and orientation with respect to the flow streamlines. Note that the time the capsule takes to realign itself with the shear plane is quite long and increases with the initial distance of the capsule tip from the shear plane as shown for example in Fig. 2.

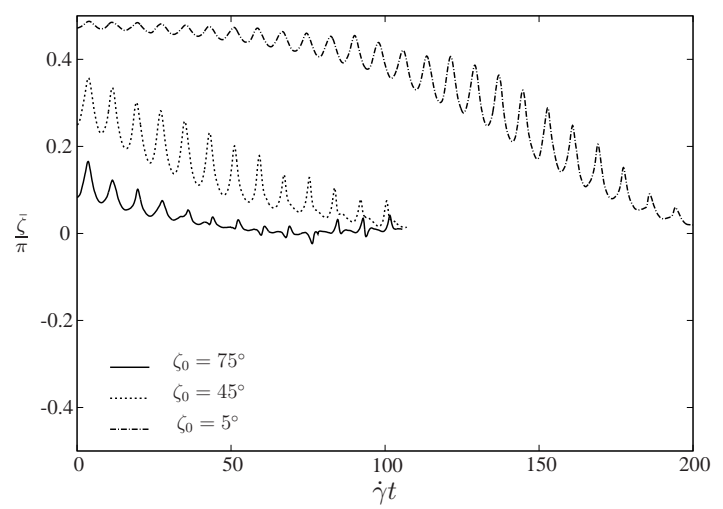

Fig. 2: Time evolution of the angle $\bar{\zeta}$ between the capsule small axis $O M_{3}^{t}$ and the shear plane for three initial orientations $\zeta_{0}$ at $C a=0.01$ $(\lambda=1)$. 
When $C a$ is further increased $(C a \geq 0.9)$, the principal axes, which were in the shear plane, exhibit small oscillations both about and within the shear plane: the equilibrium state of the capsule thus experiences a transition from a swinging to a swinging-oscillating motion, where the point $M_{3}^{0}$ precesses around the vorticity axis with a constant mean inclination. Then, for $C a>1.1$, the point $M_{3}^{0}$ converges towards the vorticity axis and the capsule takes a rolling motion that is identical to the one observed when the short axis is initially aligned with the vorticity axis $\left(\zeta_{0}=0^{\circ}\right)$. The membrane tank-treads around a steady shape as shown in Fig. 4. This motion was also observed by Omori et al. (2012) for $C a=1$. Note that when the capsule is initially positioned with $\zeta_{0}=0^{\circ}$, the rolling motion is in principle a solution of the problem for any value of $C a$. However, the present results demonstrate that this rolling motion is unstable for $C a \leq 1.1$.

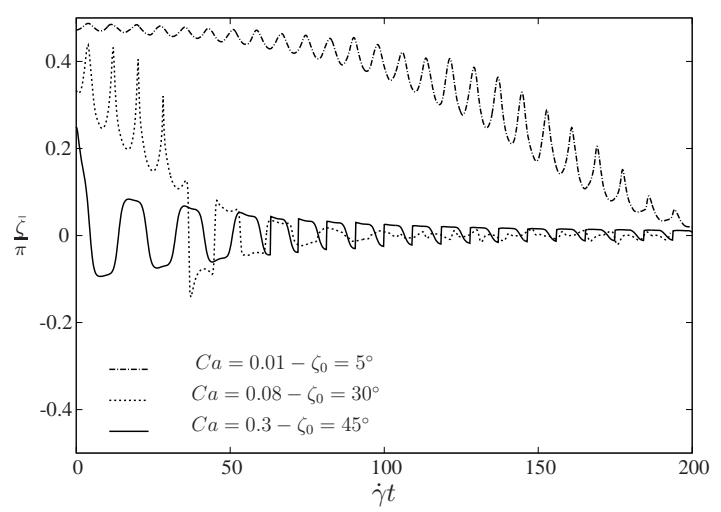

Fig. 3: Time evolution of the angle $\bar{\zeta}$ between the capsule small axis $O M_{3}^{t}$ and the shear plane for different values of $\mathrm{Ca}$ and initial orientation $(\lambda=1)$.

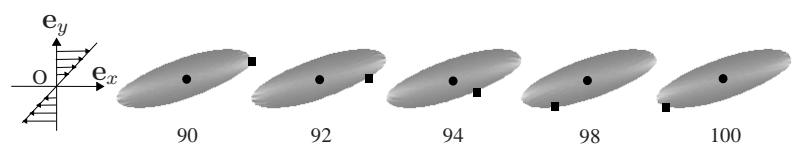

Fig. 4: Rolling motion over one half period at steady state for $C a=1.2$ and $\zeta_{0}=85^{\circ}(\lambda=1)$. The points $M_{1}^{0}(\bullet)$ and $M_{3}^{0}(\bullet)$ were initially at the tip of a long and the short axis respectively (Fig. 1)

In conclusion, for $\lambda=1$ and depending on $C a$, the capsule aligns its axis in the shear plane where it takes a quasi-solid tumbling mo- tion $(\mathrm{Ca}<0.02)$, a transition motion $(\mathrm{Ca} \in$ $[0.02,0.05])$ and a swinging motion $(\mathrm{Ca}<0.9)$. For higher values of $\mathrm{Ca}$, the capsule starts moving out of the shear plane and finally, for $C a \geq$ 1.1 , it aligns its axis with the vorticity direction and takes a rolling motion.

As the viscosity ratio increases, the $\mathrm{Ca}$ limits of the different regimes evolve with $\lambda$ (Fig. $5)$ :

- The minimum value of $C a$ for which the rolling motion is mechanically stable, decreases.

- The transition between the tumbling and swinging motions in the shear plane appears for higher $C a$.

- For $\lambda>5$, the swinging motion completely disappears and the mechanical equilibrium states are only the swinging and the rolling motions.

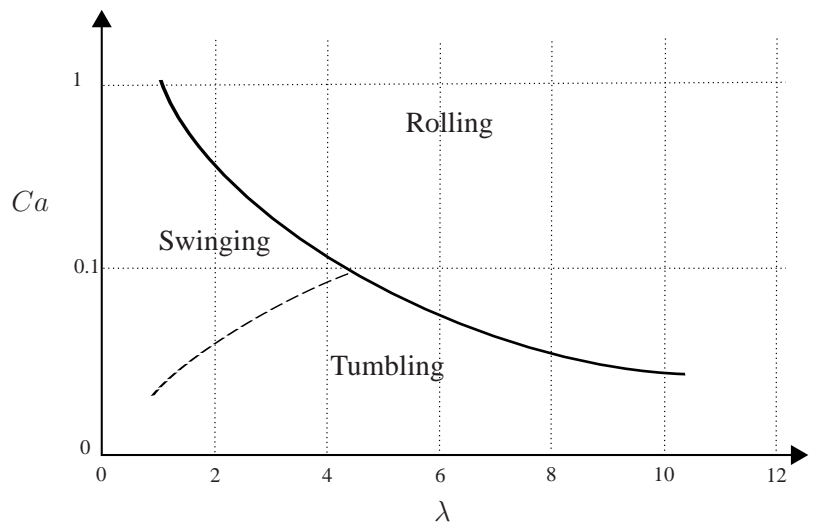

Fig. 5: Capsule shape evolution over one half period at steady state for $C a=1.2$ and $\zeta_{0}=$ $85^{\circ}(\lambda=1)$.

\section{Conclusion}

We have investigated the motion of an oblate capsule in shear flow and found that it depends on the flow strength and the viscosity of the capsule internal liquid as compared to the suspending one, but not on the initial inclination. We have shown that oblate capsules may exhibit only three possible equilibrium states (tumbling, swinging, rolling). There is therefore no procession motion at equilibrium. It is important to note that the time required to reach an equilibrium state is quite large. For 
$\lambda=1$, it is of order $\dot{\gamma} t=200$ and can increase significantly when $\lambda$ increases. In practice, the time window to observe the motion of artificial capsules or red blood cells experimentally is between $10 \mathrm{~s}$ and $50 \mathrm{~s}$ (Abkarian et al, 2007, 2008). At low shear rates, the capsules move slowly, which facilitates the recording of their dynamics in a shear flow. However, the nondimensional time of observation $\dot{\gamma} t$ is too small for the capsules to reach the mechanical equilibrium state: only the transition motion can be observed in vitro at low shear rates. When the shear rate is increased, the time window $\dot{\gamma} t$ becomes larger. Thus the higher the shear rate, the easier it becomes to design experiments where equilibrium is reached. The price to pay is that the capture of capsule dynamics is more difficult and requires even more sophisticated visualization means than for low shear experiments.

\section{Acknowledgments}

This research was funded by Ecole Polytechnique (PhD scholarship), the Conseil Régional de Picardie (MODCAP grant), by the French Ministère de la Recherche (Pilcam2 grant) and by the French Agence Nationale de la Recherche (CAPSHYDR grant ANR-11-BS09013, Labex MS2T ANR-11-IDEX-0004-02).

\section{References}

Cordasco, D., Bagchi, P., 2013. Orbital drift of capsules and red blood cells in shear flow. Phys. Fluids 25, 091902.

Dupont, C., Salsac, A.V., Barthès-Biesel, D., 2013. Off-plane motion of a prolate capsule in shear flow. J Fluid Mech. 721, 180-198.

Foessel, E., Walter, J., Salsac, A.V., BarthèsBiesel, D., 2011. Influence of internal viscosity on the large deformation and buckling of a spherical capsule in a simple shear flow. J Fluid Mech 672, 477-486.

Omori, T., Imai, Y., Yamaguchi, T., Ishikawa, T., 2012. Reorientation of a nonspherical capsule in creeping shear flow. PRL 108, 138102(5).

Skalak, R., Tozeren, A., Zarda, R.P., Chien, S., 1973. Strain energy function of red blood cell membranes. Biophys. J . 13, 245-264.

Sui, Y., Low, H.T., Chew, Y.T., Roy, P., 2008. Tank-treading, swinging, and tumbling of liquid-filled elastic capsules in shear flow. PRE 77, 016310.

Walter, J., Salsac, A.V., Barthès-Biesel, D., 2011. Ellipsoidal capsules in simple shear flow: prolate versus oblate initial shapes. J Fluid Mech 676, 318-347.

Walter, J., Salsac, A.V., Barthès-Biesel, D., Le Tallec, P., 2010. Coupling of finite element and boundary integral methods for a capsule in a Stokes flow. Int $\mathbf{J}$ Numer Methods Eng 83, 829-850. 